\title{
INTERLACING POLYNOMIALS
}

\author{
CHARLES R. JOHNSON
}

\begin{abstract}
Let $A$ be an $n$-by- $n$ Hermitian matrix. We note that the set of all monic, degree $n-1$ polynomials whose roots interlace the eigenvalues of $A$ is exactly the classical field of values of $\operatorname{adj}(\lambda I-A)$.
\end{abstract}

Let $p$ be a polynomial of degree $n$ with real roots $\lambda_{1} \leq \lambda_{2} \leq \cdots \leq \lambda_{n}$. If $q$ is a degree $n-1$ polynomial with real roots $\mu_{1} \leq \mu_{2} \leq \cdots \leq \mu_{n-1}$, we say that the roots of $q$ interlace those of $p$ if

$$
\lambda_{1} \leq \mu_{1} \leq \lambda_{2} \leq \mu_{2} \leq \cdots \leq \mu_{n-1} \leq \lambda_{n},
$$

and, in this event, we call $q$ an interlacing polynomial for $p$. We denote by $\operatorname{Int}(p)$ the set of all monic degree $n-1$ interlacing polynomials for a given degree $n$ polynomial $p$ with real roots.

OBSERVATION. The set $\operatorname{Int}(p)$ is convex.

PROOF. If $\lambda$ is a root of $p$ of multiplicity $k$, then $\lambda$ must be a root of multiplicity at least $k-1$ of each $q \in \operatorname{Int}(p)$. So, we assume, without loss of generality, that the roots of $p$ are

$$
\lambda_{1}<\lambda_{2}<\cdots<\lambda_{n} .
$$

In this event, for $1 \leq i \leq n-1$, either

$$
q\left(\lambda_{i}\right) \geq 0 \text { and } q\left(\lambda_{i+1}\right) \leq 0 \text { for all } q \in \operatorname{Int}(p)
$$

or

$$
q\left(\lambda_{i}\right) \leq 0 \text { and } q\left(\lambda_{i+1}\right) \geq 0 \text { for all } q \in \operatorname{Int}(p) .
$$

In either event, any convex combination of two polynomials in $\operatorname{Int}(p)$ must be monic and have a zero in the interval $\left[\lambda_{i}, \lambda_{i+1}\right]$, and thus lie in $\operatorname{Int}(p)$.

For an $n$-by- $n$ matrix $A$, the (classical) field of values of $A$ is defined (see [2] for a survey) by

$$
F(A)=\left\{x^{*} A x: x^{*} x=1, x \in C^{n}\right\} .
$$

Note that if the entries of $A$ are polynomials over $C$ in $\lambda$, then the elements of $F(A)$ are also polynomials in $\lambda$. Also let $A_{i}$ denote the $(n-1)$-by- $(n-1)$ principal submatrix of $A$ resulting from deletion of row and column $i$. It is well known that $F(A)$ is convex and that $F\left(A_{i}\right) \subseteq F(A)$ for a complex matrix $A$. Finally for a square matrix $A$, let $p_{A}(\lambda)=\operatorname{det}(\lambda I-A)$, the characteristic polynomial of $A$.

If $A$ is an $n$-by- $n$ Hermitian matrix, it is well known, e.g. [1], that each $p_{A_{i}} \in$ $\operatorname{Int}\left(p_{A}\right), i=1, \ldots, n$. It is equally well known that $(1 / n) p_{A}^{\prime} \in \operatorname{Int}\left(p_{A}\right)$, where $p_{A}^{\prime}$

Received by the editors April 17, 1986.

1980 Mathematics Subject Classification (1985 Revision). Primary 15A42, 15A57, 26C10; Secondary 12D10, 52A 40 .

- This work was supported in part by National Science Foundation grant DMS 8500372 and by Office of Naval Research contract number N00014-86-K0012. 
denotes the derivative of $p_{A}$. (Since $p_{A}^{\prime}=\sum_{i=1}^{n} p_{A_{i}}$, the latter fact follows from the former and the convexity of $\operatorname{Int}\left(p_{A}\right)$.)

For an $n$-by- $n$ matrix $A$, let $A(\lambda)=\operatorname{adj}(\lambda I-A)$, so that the diagonal entries of $A(\lambda)$ are polynomials of degree $n-1$ in $\lambda$, the $p_{A_{i}}(\lambda)$, and the off-diagonal entries are polynomials of degree at most $n-2$ in $\lambda$. Note that, for $e_{i}$ the $i$ th standard basis vector, $e_{i}^{T} A(\lambda) e_{i}=p_{A_{i}}(\lambda) \in \operatorname{Int}\left(p_{A}\right)$ if $A$ is Hermitian. It has also been noted [3] in connection with graph theory that $(1 / n) e^{T} A(\lambda) e \in \operatorname{Int}\left(p_{A}\right)$, for $e=(1, \ldots, 1)^{T}$, and it can be shown that there is an $x \in C^{n}, x^{*} x=1$, such that

$$
x^{*} A(\lambda) x=(1 / n) \operatorname{Tr}(A(\lambda))=(1 / n) p_{A}^{\prime}(\lambda) \in \operatorname{Int}\left(p_{A}\right) .
$$

In each case an element of $F(A(\lambda))$ is a polynomial in $\operatorname{Int}\left(p_{A}\right)$ for $A$ Hermitian. Note that $\left(1 / x^{*} x\right) x^{*} A(\lambda) x$ is a monic polynomial of degree $n-1$ in $\lambda$ in general for $0 \neq x \in C^{n}$.

In order to characterize the interlacing polynomials for a given Hermitian matrix and note that they all arise in essentially the same way, our goal is to make the following observation.

THEOREM. If $A$ is an $n$-by-n Hermitian matrix, then

$$
\operatorname{Int}\left(p_{A}(\lambda)\right)=F(\operatorname{adj}(\lambda I-A)) \text {. }
$$

ProOF. We first make some simple observations which are easily checked for any $n$-by- $n$ complex matrix $A$.

$$
\begin{gathered}
p_{S^{-1} A S}(\lambda)=p_{A}(\lambda), \quad S \text { nonsingular; } \\
\operatorname{adj}\left(\lambda I-S^{-1} A S\right)=S^{-1} \operatorname{adj}(\lambda I-A) S, \quad S \text { nonsingular; } \\
F\left(U^{*} \operatorname{adj}(\lambda I-A) U\right)=F(\operatorname{adj}(\lambda I-A)), \quad U \text { unitary; }
\end{gathered}
$$

each polynomial in $F(\operatorname{adj}(\lambda I-A))$ is monic of degree $n-1$.

Now, suppose $A$ is $n$-by- $n$ Hermitian with eigenvalues $\lambda_{1} \leq \lambda_{2} \leq \cdots \leq \lambda_{n}$ and let $U$ be unitary so that

$$
U^{*} A U=D=\operatorname{diag}\left(\lambda_{1}, \ldots, \lambda_{n}\right) .
$$

By (2) and (3) $F(A(\lambda))=F(D(\lambda))$, and by (1), $\operatorname{Int}\left(p_{A}\right)=\operatorname{Int}\left(p_{D}\right)$. So, we may assume, without loss of generality throughout, that $A$ is diagonal (i.e. $A=D$ ). In this event

$$
A(\lambda)=\operatorname{diag}\left(\prod_{j \neq 1}\left(\lambda-\lambda_{j}\right), \ldots, \prod_{j \neq n}\left(\lambda-\lambda_{j}\right)\right) .
$$

It is obvious that

$$
\text { each polynomial } \prod_{j \neq i}\left(\lambda-\lambda_{j}\right) \in \operatorname{Int}\left(p_{A}\right), \quad i=1, \ldots, n .
$$

To verify that $F(\operatorname{adj}(\lambda I-A)) \subseteq \operatorname{Int}\left(p_{A}\right)$, let $x \in C^{n}$ with $x^{*} x=1$. Then

$$
x^{*}(\operatorname{adj}(\lambda I-A)) x=\sum_{i=1}^{n} \bar{x}_{i} x_{i} \prod_{j \neq i}\left(\lambda-\lambda_{j}\right) \in \operatorname{Int}\left(p_{A}\right)
$$

because of (5) and (6) and the fact that $\operatorname{Int}\left(p_{A}\right)$ is convex. 
For the inclusion $\operatorname{Int}\left(p_{A}\right) \subseteq F(\operatorname{adj}(\lambda I-A))$, we show by induction on $n$ that every set of roots which interlace the eigenvalues of $A$ occurs as the roots of a polynomial in $F(A(\lambda))$. This is easily checked for $n=2$; suppose it has been verified through $n-1$. Suppose that $\mu_{1} \leq \cdots \leq \mu_{n-1}$ such that

$$
\lambda_{1} \leq \mu_{1} \leq \lambda_{2} \leq \mu_{2} \leq \cdots \leq \mu_{n-1} \leq \lambda_{n}
$$

are given. By the induction hypothesis there is an $x \in C^{n}$, with $x_{n-1}=0$ and $x^{*} x=1$, such that the degree $n-1$ monic polynomial $p_{x}(\lambda)=x^{*} A(\lambda) x$ has $\mu_{1}, \ldots, \mu_{n-2}$ among its roots and there is a $y \in C^{n}$, with $y_{n}=0$ and $y^{*} y=1$, such that the degree $n-1$ monic polynomial $p_{y}(\lambda)=y^{*} A(\lambda) y$ also has $\mu_{1}, \ldots, \mu_{n-2}$ among its roots. Note that the remaining root of $p_{x}$ is $\lambda_{n-1}$ and of $p_{y}$ is $\lambda_{n}$ and that

$$
p_{x}(\lambda)=\sum_{i=1}^{n} \bar{x}_{i} x_{i} \prod_{j \neq i}\left(\lambda-\lambda_{j}\right)
$$

while

$$
p_{y}(\lambda)=\sum_{i=1}^{n} \bar{y}_{i} y_{i} \prod_{j \neq i}\left(\lambda-\lambda_{j}\right) .
$$

Let $p_{\alpha}=(1-\alpha) p_{x}+\alpha p_{y}$, so that $p_{0}=p_{x}$ and $p_{1}=p_{y}$, while $\mu_{1}, \ldots, \mu_{n-2}$ are roots of the degree $n-1$ monic polynomial $p_{\alpha}$ for all scalars $\alpha$. As $\alpha$ varies from 0 to 1 , the remaining root of $p_{\alpha}$ varies continuously from $\lambda_{n-1}$ to $\lambda_{n}$. Let $\alpha_{0}, 0 \leq \alpha_{0} \leq 1$, be such that $p_{\alpha_{0}}\left(\mu_{n-1}\right)=0$ and let

$$
w_{i}=\left[\left(1-\alpha_{0}\right) \bar{x}_{i} x_{i}+\alpha_{0} \bar{y}_{i} y_{i}\right]^{1 / 2}, \quad i=1, \ldots, n,
$$

and $z_{i}=w_{i} /\left(w^{*} w\right)^{1 / 2}$. Then $p_{z}(\lambda)=z^{*} A(\lambda) z \in F(A(\lambda))$ is the element of $\operatorname{Int}\left(p_{A}\right)$ with roots $\mu_{1}, \ldots, \mu_{n-1}$.

REMARK. Since $p_{A_{i}}(\lambda)=e_{i}^{T} \operatorname{adj}(\lambda I-A) e_{i} \in F(A(\lambda))$, the classical interlacing inequalities for a Hermitian matrix follow from the theorem. As the classical interlacing inequalities were not used in the proof, a simple and self-contained demonstration of them is contained therein.

The sufficiency of the interlacing inequalities for a principal submatrix of a Hermitian matrix also follows from the theorem, as any element of $F(\operatorname{adj}(\lambda I-A))$ appears on the diagonal of an appropriately chosen unitary similarity.

REMARK. It follows from the theorem that for an $n$-by-n Hermitian matrix $A, F(\operatorname{adj}(\lambda I-A))$ is convex, generalizing slightly the familiar fact that $F(B)$ is convex for an $n$-by- $n$ complex matrix $B$. If $B(\lambda)$ is a general polynomial matrix, is $F(B(\lambda))$ convex?

REMARK. It also follows from the above observations that $\operatorname{Int}(p)$ is the convex hull of

$$
\left\{\prod_{j \neq 1}\left(\lambda-\lambda_{j}\right), \ldots, \prod_{j \neq n}\left(\lambda-\lambda_{j}\right)\right\},
$$

where $\lambda_{1} \leq \lambda_{2} \leq \cdots \leq \lambda_{n}$ are the roots of $p$. 


\section{REFERENCES}

1. R. Horn and C. R. Johnson, Matrix analysis, Cambridge Univ. Press, New York, 1985.

2.

3. K. Regan, private communication.

Department of Mathematical Sciences, Clemson University, Clemson, SOUTH CAROLINA 29634-1907 